\title{
Teresa Bermúdez, figlia di Bermudo II: Una biografia storiografica
}

Marco Alvise Meneghetti Friedrich-Alexander Universität Erlangen-Nürnberg Centre de Recherches Historiques de l'Ouest (CERHIO-Angers), CNRS UMR 6258 marco.meneghetti@fau.de 
Resumen: De la infanta Teresa, hija del rey leonés Bermudo II, tenemos pocas informaciones históricas. Se la conoce también por su matrimonio con un "rey musulmán”, aunque, según la historiografía moderna, esto es por lo menos dudoso. Nos queda la transcripción de su inscripción funeraria, que hasta el siglo XVIII se encontraba en el monasterio de San Pelayo de Oviedo, donde murió, que es lo más parecido que tenemos a la narración de su vida si no tenemos en cuenta el episodio

Aвstract: Little is known about Teresa Bermúdez, daughter of Bermudo II of León. Some medieval chronicles, written in the Iberian Peninsula, tell us about her alleged marriage with a Muslim king of Toledo, while others believe this was al-Mansur (or Almanzor), a Muslim military chief, after whose death she would have come back to León, ending her life in the monastery of St. Pelagius in Oviedo, a well-known place in medieval times often linked with the infantas of the kingdom (according to a legend, it was founded by king Alfonso II of Asturias, and Velasquita, the first wife of Bermudo II, is also known to have lived there). The main narrative about this event is provided by the Chronicle of Pelagius, bishop of Oviedo, which tells the deeds of the Leonese kings from the age of Bermudo II (985-999) to the death of Alfonso VI (1072-1109), and was later reprised by the Chronicle of Nájera and the "General Histories" of the 13th century.

Furthermore, a few documents have been linked to Teresa, as well as her funerary inscription, which has been lost in the 18th century but of which we possess the transcription; it could have been made slightly later, but it gives us a date for the death (and, surprisingly, the time at de su casamiento. Este artículo intenta reconstruir lo que sabemos sobre ella, y de dar una visión completa sobre los hechos (reales o legendarios) vinculados a este personaje, a través de una panorámica que tenga en cuenta de todo lo que ha sido escrito sobre Teresa al interior de la historiografía moderna y contemporánea.

Palabras clave: Teresa Bermúdez, Bermudo II, Almanzor, crónicas medievales, Memoria.

which it allegedly happened) of the princess-turned-nun. Interestingly, another inscription, dedicated to another nun with the same name, has been found during the excavations of the church of San Salvador in Oviedo (now the Cathedral): it has been tried to identify her with the Bermudo's daughter, but, given the different dates of death reported by both texts, it is almost impossible to trace a connection between them.

Many doubts about the marriage and the existence of Teresa itself were lifted by modern and contemporary historiography, which has tried to define it chronologically and historically, and it has even supposed that her epitaph was later made to provide evidence for the story of Pelagius of Oviedo: this article tries to give an historical perspective about what is certain about the princess and what has been written about her in modern and contemporary works; the need for a sort of "biographical history" for some of the lesser known characters in Spanish medieval history is here also stressed.

KeYwords: Teresa Bermúdez, Bermudo II, Almanzor, medieval chronicles, Memory. 
$\mathrm{N}$ egli ultimi tempi, lo studio della storia medievale iberica ha conosciuto un notevole sviluppo grazie alla diffusione di quelle che potremmo definire "biografie regali": articoli, o, spesso, interi testi monografici, dedicati all'esplorazione della vita e delle imprese di uno specifico personaggio attraverso le testimonianze narrative o documentarie da lui lasciate nel corso dei secoli. Nella maggior parte dei casi, si è scelto, a causa della maggiore disponibilità di materiale maneggiabile, di concentrarsi su re ed (in alcuni casi) regine, $o$, più genericamente, personaggi d'alto rango nelle corti dei regni fioriti in quei secoli nella Penisola iberica.

A mio parere, l'inestimabile patrimonio cronachistico dell'Edad Media española consente però di lanciarsi anche nello studio di altre figure, a torto considerate "di seconda schiera”, il cui ricordo ci è stato tramandato, seppur in maniera frammentaria od oscura, dalle fonti. Un elemento molto interessante per la realizzazione di un simile ritratto può peraltro contare su quanto scritto (molto o poco che sia) dalla storiografia precedente, dimensione che non fa che aggiungere ulteriori sfumature ed opinioni con le quali lo storico può confrontarsi. Il mondo femminile, nella sua dimensione ispanica ma non solo, resta uno dei versanti più stimolanti ed inesplorati (se guardiamo al di là della manciata di sovrane e nobildonne più note, di cui la regina Urraca è forse l'esempio più lampante') in tal senso.

\footnotetext{
${ }^{1}$ Naturalmente mi riferisco al fondamentale testo di B. F. Reilly, The Kingdom of León-Castilla under Queen Urraca, IIO9-II26, New York, 1986; si vedano inoltre i lavori di Therese Martin, atti a sottolinere l'importanza di Urraca in quanto sovrana "regnante" (reigning queen), qualità che la
}

\section{TERESA BERMÚDEZ ED IL SUO PRESUNTO MATRIMONIO}

Del regno di Bermudo II, re di León dal 985 al 999, ci sono giunti numerosi resoconti, contenuti nelle cronache medievali iberiche: quelli di Sampiro ${ }^{2}$, vescovo di Astorga (incorporata nella cosiddetta Historia Silensis o Legionensi $^{3}$ ) e Pelayo di Oviedo (che a sua volta, nella sua controversa opera di ricompilazione storica, il Corpus Pelagianum, incluse una versione del testo di Sampiro fortemente interpolata; lo stesso Pelayo scrisse una cronaca che va dall'ascesa di Bermudo II nel 985 sino alla morte di Alfonso VI, occorsa nel $1109^{4}$ ), nella Chronica Naierensis ${ }^{5}$, nonché nelle cosiddette sto-

differenzia notevolmente dalle regine consorti del suo tempo: T. Martin, "The Art of a Reigning Queen as Dynastic Propaganda in Twelfth-Century Spain", Speculum, 2005, vol.80, pp. II34-II71; T. Martin, Queen as King: Politics and Architectural Propaganda in Twelfth-Century Spain, Leiden, 2006.

${ }^{2}$ J. Pérez de Urbel (ed.), Sampiro, su crónica y la monarquía leonesa en el siglo X, Madrid, 1952.

3 J. Pérez de Urbel, A. Ruiz Zorrilla (ed.), Historia Silense, Madrid, 1959. inoltre, la traduzione Introducción a la Historia Silense con versión castellana de la misma y de la crónica de Sampiro, (traduzione a cura di M. Gómez Moreno), Madrid, I92I possiede un buon apparato critico all'opera.

${ }^{4}$ B. Sánchez Alonso (ed.), Crónica del Obispo Don Pelayo, Madrid, 1924. l'insieme testuale e cronístico realizzato dal prelato ovetense viene esaminato da E. Jérez, "Arte compilatoria pelagiana. La formación del Liber cronicorum", in A. Arizaleta (ed.), Poétique de la chronique. L'écriture des textes historiographiques au Moyen Âge (Péninsule Ibérique et France), Toulouse, 2008, p. 47-87; si veda inoltre R. Alonso Álvarez, "El obispo Pelayo de Oviedo (IIOI-II53): historiador y promotor de códices iluminados", Semata. Ciencias Sociais e Humanidades, vol. 22, 2010, pp. 331-350.

${ }_{5}^{5}$ Per il presente articolo mi sono avvalso dell'edizione A. Ubieto Arteta (ed.), Crónica Najerense, Zaragoza, 1985. Si veda anche la più recente J. A. Estévez Sola (ed.), Chronica Hispana saeculi XII, Pars II: Chronica Naierensis (CC CM, LXXI A), , Turnhout, 1995. 
rie generali più tarde, come quelle dei primati Jiménez de Rada $^{6}$ e Luca di Tuy e quella, largamente ispiratasi ad esse, di re Alfonso X el Sabio ${ }^{8}$.

La parte del testo pelagiano dedicata a questo monarca è tuttavia, probabilmente, la più notevole, in quanto contenente alcuni dati molto interessanti sulla figlia avuta dal secondo matrimonio con Elvira García (figlia del conte castigliano García Fernández): Teresa. A lei, il vescovo di Oviedo dedica alcune righe significative, affermando come, alla morte del padre, essa fosse stata data in sposa ipsa nolente dal fratello, l'appena incoronato re Alfonso V (regnante dal 999 al 1028), al sovrano musulmano di Toledo, allo scopo di mantenere la pace tra i due territori'.

Secondo la narrazione, una volta giunta presso la corte dell'infedele Teresa ingiunse a questi di non toccarla, al fine di non incorrere nell'ira divina (Noli me tangere, quia paganus es; si vero me tetigeris Angelus Domini interficiet te); ma il potente personaggio, ignorando totalmente le sue preghiere, concubit cum ea semel, et statim, percussus est ab Angelo Domini ${ }^{10}$. Terrorizzato dall'accaduto, e moribondo a causa del castigo ricevuto, il sovrano fece preparare oro ed argento, tessuti pregiati e gemme e, convocata Teresa, la rimandò carica di doni alla corte del fratello, dove ella prese l'abito monacale e, trasferitasi nel monastero di San Pelayo

\footnotetext{
${ }^{6}$ J. Fernández Valverde (ed.), Roderici Ximenii de Rada Historia de rebus Hispanie, sive, Historia Gothica (CC CM, LXXII), J. Fernández Valverde (ed.), Turnhout, 1987; si veda anche la traduzione in castigliano, Rodrigo Jiménez de Rada, Historia de los hechos de España, (traduzione a cura di J. Fernández Valverde), Madrid, 1989.

${ }^{7}$ E. Falque Rey (ed.), Lucae Tudensis Chronicon Mundi (CC CM, LXXIV), Turnhout, 2003. Si veda anche J. Puyol (ed.), Crónica de España por Lucas, obispo de Tuy. Primera edición del texto romanceado, Madrid, 1926.

${ }^{8}$ R. Menéndez Pidal (ed.), Primera crónica general: estoria de Espańa que mandó componer Alfonso el Sabio y se continuaba bajo Sancho IV en I289, Madrid, 1906 .

${ }^{9}$ Ne riporto qui il testo: Predictus etiam princeps habuit duas legitimas uxores. Una nomine Velasquitam, quam viventem dimisit; aliam domine Geloira duxit uxorem, ex qua genuit duos filios, Adefonsum et Tarasiam. Ipsam vero Tarasiam post mortem patris sui dedit frater eius Adefonsus in coniugio, ipsa nolente, cuidam pagano regi toletano pro pace. Ipsa autem, ut erat christiana, dixit pagano regi: Noli me tangere, quia paganus es; si vero me tetigeris Angelus Domini interficiet te. Tunc rex derisit eam, et concubit cum ea semel, et statim, sicut predixerat, percussus est ab Angelo Domini. Ille autem ut sensit mortem propinquam adesse sibi, vocavit cubicularios et consiliarios suos, et precepit illis onerare camellos auro et argento, gemmis et vestibus preciosis, et adducere illam ad Legionem cum totis illis muneribus. Quo loco illa in monachali habitu diu permansit, et postea in Obeto obiit, et in Monasterio Sancti Pelagii sepulta fuit. (Crónica del Obispo Don Pelayo, cit., pp. 63-65).

${ }^{10}$ Id., p. 64.
}

ad Oviedo, vi morì e vi venne sepolta ${ }^{11}$. La vicenda è inclusa anche all'interno della Chronica Naierensis, peraltro a sua volta realizzata con frammenti della cronaca di Pelayo e di altri testi storiografici anteriori ${ }^{12}$.

A tale racconto vanno aggiunti altri dettagli, espressi da Luca di Tuy, che afferma come, trovandosi ancora Alfonso sotto tutela dei notabili del regno a causa della minore età (aveva infatti soltanto cinque anni al momento della morte del padre), la decisione fosse stata presa da questi ultimi, lasciando inoltre sottintendere come ciò fosse avvenuto in buona fede, a causa del fingersi cristiano da parte del re islamico, peraltro in procinto di attaccare il reame leonese ${ }^{13}$.

La storia della sventurata Teresa venne narrata già da Ambrosio de Morales nella sua Coronica ${ }^{14}$ e di nuovo nel secolo XVIII da Manuel Risco, che nel trentacinquesimo tomo dell'España Sagrada, il secondo dedicato alla città e alla diocesi di León, afferma anche come lo sposo avesse nome "Abdalá", probabile trascrizione castiglianizzata del nome arabo Abd-Allah impiegata per la prima volta dal Tudense ${ }^{15}$.

Già Reinhart Dozy, a metà del diciannovesimo secolo, espresse alcuni dubbi su tale vicenda, pur riconoscendone la veridicità storica di fondo ${ }^{16}$. Lesistenza di Teresa è fuor di dubbio, confermata da almeno quattro diplomi che recano la sua firma, il primo dell'anno 1017, il secondo datato 1 marzo 1028 ed il terzo 27 gennaio 1030 (tutti a beneficio del clero di Compostela). ${ }^{17}$, ed il quarto, ovetense, del 22 dicembre $1037^{18}$, e dall'iscrizione funeraria che la riguarda, della quale parleremo in seguito.

\footnotetext{
${ }^{11}$ Id., p. 65; a pagina 66 è riportato l'episodio della traslazione delle spoglie di San Pelayo da León a Oviedo, allo scopo di proteggerle dalle razzie compiute da Almanzor, nel 994.

${ }_{12}$ Crónica Najerense, cit., p. 83; le differenze testuali con Pelayo sono davvero poche, e si limitano perlopiù all'impiego di una mera serie di sinonimi o modifiche ortografiche.

${ }^{13}$ Lucas de Tuy, Crónica de España, cit., pp. 332-335.

${ }^{14}$ A. de Morales, Coronica general de Espańa que continuaba Ambrosio de Morales coronista del rey nuestro seńor Don Felipe II, VIII, Madrid, I79I, pp. 442-443.

${ }^{15}$ M. Risco, ES, t. XXXV: León, Madrid, I784, pp. 15-16.

${ }^{16}$ R. Dozy, Recherches sur l'histoire et la littérature de l'Espagne pendant le Moyen-Âge (2 voll.), 2 ${ }^{\mathrm{a}} \mathrm{ed}$, Leiden, I860, I, pp. 2OI-2IO.

17 I due diplomi compostellani sono editi all'interno di M. Lucas Álvarez (ed.), Tumbo A de la Catedral de Santiago, Santiago de Compostela, 1998 , diplomi n. 90, 93 e 94. Teresa appare anche nelle sottoscrizioni ai diplomi $\mathrm{n}$. 66 (30 dicembre I028) e 67 (25 agosto I032).

${ }^{18}$ Id., pp. 202-203; il documento di Oviedo venne citato per la prima volta da P. de Sandoval, Historia de los reges de Castilla y León, Pamplona, I634, f. $54 \mathrm{r}$
} 
Una notizia curiosa riguarda il suo ritratto nel cartulario compostellano, dove, secondo quanto riporta lo stesso Dozy, elle porte un sceptre et une couronne, chiara allusione, a detta dell'autore, al suo status di moglie di un sovrano musulmano ${ }^{19}$ : tuttavia, quell'immagine femminile rintracciabile nel Tumbo A della Cattedrale di Santiago è stata da tempo attribuita alla regina Urraca, contraddicendo così l'ipotesi dell'autore ${ }^{20}$. Vi è però un'altra immagine. presente nel folio $38 \mathrm{v}$ del "Tumbo A", segnalata da Lucas Álvarez come raffigurante "Sancha y Teresa, hijas de Vermudo II"21.

Secondo lo studioso olandese, il diploma del 1017 prova come in quell'anno Teresa fosse già tornata in seno alla propria famiglia; ma il primo sovrano musulmano indipendente di Toledo, salito alla ribalta in seguito alla furibonda guerra civile scoppiata all'inizio del secolo XI nel califfato di Córdoba, morì nel 1036, vale a dire quasi vent'anni dopo ${ }^{22}$. Il re di Toledo, inoltre, era un personaggio di secondo piano, e dargli in moglie la propria sorella avrebbe significato, per Alfonso $\mathrm{V}$, in qualche modo sottomettervisi senza alcun profitto apparente ${ }^{23}$.

Ecco dunque che la chiave potrebbe risiedere altrove: per la precisione, in Muhammad ibn Abi Amir al-Mansur, meglio noto alla storiografia di lingua castigliana come Almanzor, il potentissimo caudillo e ciambellano di Hisham II spesso definito come "il califfo nell'ombra" ${ }^{24}$, responsabile di una lunga serie di campagne militari contro il Regno di León nell'ultimo quarto del secolo X (e talvolta confuso dalle fonti cristiane medievali con il vero sovrano cordobese). Per trovare una notizia in appoggio a tale ipotesi, è necessario lasciare per un momento la storiografia leonese e volgerci alle fonti arabe: per la precisione, ad Ibn Khaldun, autore attivo sul finire del Trecento che registra come nell'anno 993 Bermudo II avesse inviato una

\footnotetext{
${ }^{19}$ Id., pp. 202-203.

${ }^{20}$ Sulla rappresentazione delle infante regie nei cartulari medievali iberici si veda G. Fournès, "Iconologie des infantes (Tumbo A et Tumbo B de la cathédrale de Saint-Jacques de Compostelle et Tumbo de Touxos Outos)", e-Spania [online], 5 giugno 2008, pubblicato il I febbraio 2008, consultato il I2 giugno 20I4. URL: http://e-spania.revues.org/I2033.

${ }_{21}$ M. Lucas Álvarez, Tumbo A de la Catedral de Santiago, cit., p. 203; si veda anche p. 34I, n. XXII.

${ }^{22}$ Id., p. 203; qui Dozy si appoggia alle notizie di Ibn Khaldun; il sovrano in questione, Yayisch, morì probabilmente già un anno prima.

${ }^{23}$ Id., pp. 203-204.

${ }^{24}$ E' questo anche il titolo della recente ed esaustiva biografia di A. Echevarría Arsuaga, Almanzor, un califa en la sombra, Madrid, 2orI.
}

figlia presso il nemico, che, dopo averla inizialmente tenuta in schiavitù, la prese successivamente in sposa ${ }^{25}$. La morte di Almanzor, occorsa nel 1002, e la successiva inutilità di Teresa a corte, potrebbero aver spinto il di lui figlio e successore, Muzaffar, a restituirla al fratello Alfonso V, forse addirittura come condizione per il rispetto di una tregua siglata l'anno seguente tra i due ${ }^{26}$.

Tale teoria, espressa nel 1860, venne totalmente screditata nel 1903 da un articolo di Emilio Cotarelo, il quale ritenne, con vena non poco critica nei confronti di Dozy, come tutta la vicenda non avesse il minimo fondamento di verità ${ }^{27}$. Definendo la narrazione di Ibn Khaldun nient'altro che los delirios de un compilador de fines del siglo XIV ${ }^{28}$, l'Autore si lancia in una totale opera di smembramento della notizia dataci dalle fonti arabe (a suo parere valorizzate in maniera eccessiva da un manipolo di studiosi, prevalentemente arabisti, pronti a sconfessare automaticamente qualsiasi lavoro prodotto al di fuori di al-Andalus), senza, a parere di chi scrive, porre troppo in risalto come invece l'informazione sia similmente data (e con gli stessi limiti di incertezza) anche da cronisti cristiani, in primis Pelayo di Oviedo stesso, il cui punto di vista è liquidato in appena poche righe ${ }^{29}$; tuttavia, alcuni dati ivi proposti sono degni di considerazione.

Abbiamo già detto come Teresa fosse nata dal secondo matrimonio di Bermudo II, e, supponendo che ella fosse la primogenita, dobbiamo collocare la sua data di nascita precedentemente al 994 (quando nacque Alfonso V), e, dunque, tra il 991 ed il 993 al massimo ${ }^{30}$ : un lasso di tempo che pone fuori discussione la sua identificazione con la fanciulla citata da Ibn Khaldun, che egli avrebbe sposato quando la "nostra" Teresa era ancora in fasce; ed in ogni caso problematico anche ponendo più in avanti i limiti cronologici della vicen$\mathrm{da}$, tenendo conto di come, basandoci su queste medesime date, ella potesse avere, nel 1002, soltanto dieci

\footnotetext{
${ }^{25}$ R. Dozy, Recherches, cit., pp. 203-204.

${ }^{26}$ Ibid.

${ }^{27}$ «El supuesto casamiento de Almanzor con la hija de Bermudo II», in La España Moderna, I69, 1903, pp. 42-55.

${ }^{28}$ Id., p. 46.

${ }^{29}$ Id., p.44.

${ }^{30}$ J. Rodríguez Fernández, "La monarquía leonesa. De García I a Bermudo III (9IO-IO37)", El reino de León en la Alta Edad Media, III, León, I995, pp. 362-372.
} 
anni o meno ${ }^{31}$. Lo stesso Claudio Sánchez-Albornoz, in una nota a margine del suo lungo studio sull'esercito nel regno delle Asturie-León, ha dimostrato i suoi dubbi relativi all'età della principessa ${ }^{32}$.

Per quanto concerne l'enigmatica figura di "Abdalá", invece, Cotarelo propone l'identificazione con un governatore militare di Toledo coinvolto in una furiosa lotta di potere con Almanzor, dalla cui prigionia riuscì a liberarsi raggiungendo uno status di semi-indipendenza che giustificherebbe la definizione di "re" data dalle fonti ${ }^{33}$.

La vicenda resta, quindi, complessa. A partire da quell'articolo del 1903, per molti decenni sono mancati altri studi approfonditi dedicati a Teresa ${ }^{34}$, e le problematiche suscitate dall'identità del suo presunto sposo sono tutt'altro che risolte; un recente contributo, all'interno di un quadro più ampio, è però costituito dall'analisi di Simon Barton relativa ai matrimoni interreligiosi nella Spagna altomedievale, che oltre a presentare la vicenda della figlia di Bermudo II in maniera sintetica ma convincente all'interno di un contesto più generale (con inoltre il merito di gettare luce anche su altri esempi simili e meno conosciuti), riassume anche le divergenti scuole di pensiero espressesi nel corso dei decenni precedenti su tale figura ${ }^{35}$.

Sale alla memoria il caso di un'altra principessa, della quale non ci è noto neppure il nome, che tuttavia soffrì un analogo destino: la figlia di Desiderio, ultimo re dei Longobardi, data in sposa, secondo alcuni te-

\footnotetext{
${ }^{31}$ A. Echevarría Arsuaga, Almanzor, un califa en la sombra, cit., p. Io9.

32 C. Sánchez-Albornoz, "El ejército y la guerra en el reino asturleonés, 718-I037", Ordinamenti Militari in Occidente nell'Alto Medioevo ("Settimane di Studio del Centro Italiano di Studi sull'Alto Medioevo", XV), I, 1968, pp. 293-428; si veda in particolara la n. 450 a p. 420.

${ }^{33}$ E. Cotarelo: "El supuesto casamiento de Almanzor con la hija de Vermudo II", cit., p. 45; l'Autore non specifica tuttavia le sue fonti a riguardo, ed ammette come, nella cronachistica araba, di tale personaggio non vi sia traccia.

${ }^{34}$ Una citazione della vicenda è però contenuta in G. Cavero Domínguez, El monasterio medieval, sede de solar nobiliario y refugio de las mujeres de la aristocracia, in Monasterios y nobles en la España del románico: entre la devoción y la estrategia, a cura di J. A. García de Cortázar e R. Teja, Aguilar de Campoo, 20I4, pp. 97-I35: Teresa viene menzionata alle pp. IOI-IO2, dal quale sono partito per la redazione del presente articolo, oltre naturalmente alla già citata biografia di Almanzor, al lavoro di Rodríguez Fernández e a R. Barkai, Cristianos y musulmanos en la Espańa medieval (El enemigo al espejo), Madrid, 1984, p. 137, nonché ad alcuni lavori dedicati specificamente al monastero di San Pelayo di Oviedo che citerò in seguito; ringrazio la professoressa Cavero per avermi dato la possibilità di leggere il suo lavoro prima della sua messa in stampa.

${ }^{35}$ S. Barton, "Marriage across frontiers: sexual mixing, power and identity in medieval Iberia", Journal of Medieval Iberian Studies, vol. 3:I, 2005, Dell'episodio narrato da Pelayo si parla alle pp. I2-I4..
}

sti cronachistici ed annalistici, a Carlo Magno, ed in seguito, probabilmente, ripudiata poco prima dell'assalto franco alla Penisola italiana, e rientrata presso la corte pavese prima di svanire nel nulla ${ }^{36}$; ma, se la cosiddetta "Ermengarda" (così chiamata, senza alcuna base storica, da Manzoni nella sua tragedia Adelchi) fu vittima di una vera e propria damnatio memoriae condotta dalla storiografia carolingia allo scopo di occultare tale episodio alla luce degli eventi successivi, di Teresa abbiamo perlomeno alcuni elementi in più, che ne consentono di ricostruire l'identità ed il destino finale.

\section{L'ISCRIZIONE FUNERARIA DEL MONASTERO DI SAN PELAYO (OVIEDO, ASTURIE)}

Riallacciandosi al discorso fatto in apertura al presente articolo, è necessario porre in evidenza l'importanza dell'epigrafia nello studio della "storia biografica" medievale, specie quando essa costituisce uno dei pochi apporti possibili per la conoscenza di uno specifico personaggio. Anche l'impossibilità di reperire un epitaffio nella sua forma originaria, se integrata dall'esistenza di una trascrizione attendibile o da altri elementi che ne testimonino la passata esistenza, può rivelarsi utile per la ricostruzione storica e biografica di eventi passati: un insieme "tematico" di epigrafi legate da un filo logico comune può, allo stesso modo, gettare maggior luce su aspetti più vasti della storia di un territorio o di un'epoca.

Un'edizione delle iscrizioni dedicate ai membri delle élites del Regno asturleonese, ad esempio (ma non solo: si pensi ad altre entità continentali più o meno coeve, quali il Regno di Pamplona o, andando più lontano, l'Impero Carolingio od il primigenio Ducato di Normandia) contribuirebbe non poco, a mio parere, alla comprensione di aspetti che altrimenti, visti soltanto con l'ottica di casi isolati, verrebbero esaminati in maniera superficiale, oltre a fornire l'occasione di verificare l'esistenza di formule o di trattamenti testuali comuni per tali segmenti della popolazione ${ }^{37}$.

\footnotetext{
${ }^{36}$ La famiglia di re Desiderio: un percorso attraverso le fonti (2013), scaricabile in formato PDF all'indirizzo https://www.academia. edu/4421246/La famiglia di re Desiderio un percorso tra le fonti.

${ }^{37}$ E' ció che ho tentato di fare, per quanto concerne il Regno longobardo,
} 
Abbiamo già accennato all'epigrafe funeraria di Teresa, posta nel monastero di San Pelayo di Oviedo (è possibile che ella avesse vissuto in precedenza nell'omonimo cenobio sito a León, in seguito divenuto San Isidoro $^{38}$ ), del quale fu badessa, secondo Carvallo, dal 1022 sino alla morte ${ }^{39}$, ed all'interno del quale si trovava anche, curiosamente, Velasquita, moglie di Bermudo II prima della madre della stessa Teresa ${ }^{40}$ : azzardo qui la supposizione che, in occasione del passaggio delle reliquie legate a San Pelayo dalla capitale alle Asturie, anche il seguito (o perlomeno una parte consistente di esso) di monache residenti nel monastero leonese si fosse trasferito più a nord, dando continuità alle due fondazioni in una situazione di assoluta emergenza come dovette essere quella provocata dagli attacchi musulmani dell'epoca, diretti sempre più in profondità non solo del Regno di León, ma anche di quello di Pamplona e delle nascenti contee pirenaiche (basti pensare all'incursione di Almanzor occorsa nel 997 a Santiago de Compostela, luogo già all'epoca di importanza fondamentale per l'identità religiosa -e non solo- del reame e di tutto il cristianesimo peninsulare). Teresa dovette aver raggiunto il nuovo cenobio pelagiano alcuni anni dopo l'arrivo dei resti del santo in territorio asturiano, occorso nel 994 (quando, come sappiamo, ella era probabilmente ancora in fasce!), pur continuando, sino al 1030, a figurare in documenti emessi dalla corte leonese (sebbene a beneficio dell'episcopato compostellano), motivo che rende l'affermazione di Carvallo quantomeno dubbiosa.

Della lapide, andata perduta probabilmente sul finire del secolo XVIII, conserviamo la trascrizione te-

in M. Meneghetti, L'epigrafia elitaria longobarda. Formule e contenuti testuali, Tesi di Laurea presentata presso l'Università di Venezia "Ca' Foscari", 20I2; esistono tuttavia ottime raccolte di testi epigrafici su base territoriale, come lo studio di F. Diego Santos, Inscripciones medievales de Asturias, Oviedo, 1994.

${ }^{38}$ E. Cotarelo, "El supuesto casamiento de Almanzor con la hija de Vermudo II", cit., p. 50.

${ }^{39}$ L. A. de Carvallo, Antigüedades y cosas memorables del Principado de Asturias, Oviedo, 1695 (ried. Gijón, 1977), p. 290.

${ }^{40}$ L'iscrizione appare in C. M. Vigil, Asturias monumental, epigráfica y diplomática: datos para la historia de la provincia, Oviedo, 1887 (ed. facsimile, Oviedo, 1987), vol. I, p. I33. Per un'analisi delle origini del cenobio in questione, si veda l'articolo di F. J. Fernández Conde ed I. Torrente Fernández: "Los orígenes del monasterio de San Pelayo: aristocracía, poder y monacato", Territorio, Sociedad y Poder, 2 (2007), pp. 18I-202; per una ricognizione sulle fonti riguardanti il monastero si veda inoltre il volume di F.J. Fernández Conde; I. Torrente Fernández; G. de la Noval Menéndez, El monasterio de San Pelayo. Historia y Fuentes, Oviedo, 1978. stuale, che vale la pena riprodurre per intero: En quem cerni caxa tegit / compago sacra, hic dilecta Deo recubans I Tarasia Christo dicata, proles Veremundi regis I et Geloirae reginae, genere clara paren $(t a) t u(m)$, / clarior et merito vitam duxit praeclaram, / ut continet norma; hanc imitari velis / si bonus esse cupis / vel si obiit sub die (septimo) VII Klas. Magii, feria IIII / hora mediae noctis, era MLXXVII, post / peracta aetate saeculi porrecta per ordinem VI. / Da, Christe, queso veniam, parce precor. Amen. ${ }^{41}$

Oltre all'appartenenza alla famiglia reale, viene dunque segnalata una data di morte ben precisa, corrispondente al 25 aprile 1039, che permette di raggiungere un'ideale conclusione della vicenda della sfortunata principessa: quel giorno, che in quell'anno cadde effettivamente di mercoledì, è secondo César García de Castro Valdés un dato a favore dell'autenticità del manufatto, da lui ritenuto in buono stato e di facile leggibilità a causa delle scarse divergenze presenti nelle varie edizioni del suddetto ${ }^{42}$, ed inoltre la datazione proposta dai diplomi verrebbe interamente rispettata, oltre a confermare la presenza di Teresa, attraverso il diploma asturiano, ad Oviedo nel dicembre 1037, ossia soltanto poco tempo prima; tuttavia, il tono retorico impiegato nel testo potrebbe far pensare ad una data di realizzazione più tarda per l'iscrizione funebre (tali caratteristiche liriche iniziano infatti, secondo lo studioso, ad apparire in gran numero a partire dagli epitaffi del secolo XII), magari basata su di un obituario contenente la data di decesso di Teresa ed in seguito perduto ${ }^{43}$.

\section{L”ALTRA" TERESA}

E' possibile tentare un interessante parallelo tra questa testimonianza epigrafica ed un'altra, curiosamente relativa ad una donna con il medesimo nome, rinvenuta durante la campagna di scavi effettuata tra il 1940 ed il 1948 nella Cattedrale di Oviedo ed oggi custodita nel Museo ad essa annesso: il perso-

\footnotetext{
${ }^{41}$ F. Diego Santos, Inscripciones medievales de Asturias, cit., pp. I22-I23, segue la trascrizione fattane da Tirso de Áviles correggendo martii con magii.

${ }_{42}$ C. García de Castro Valdés, Arqueología cristiana de la Alta Edad Media en Asturias, Oviedo, 1995, pp. I7I-I72; si veda in particolare p. 172.

${ }^{43} \mathrm{Ibid}$.
} 
naggio in questione morì, secondo l'analisi di García de Castro Valdés, alle nove di sera del 31 dicembre 1057 (ora completa II Kalendas Ianuarias era LXLVI post millesima $)^{44}$. Una datazione così precisa, con inclusa l'ora del decesso, vera o presunta, era comune all'interno di un contesto monastico, ed infatti la riscontriamo in entrambi gli esempi; tuttavia, la maggiore sinteticità della seconda iscrizione, priva di florilegi testuali o di elogi alla figura della defunta al di là di una rapida presentazione della stessa (Obiit famula Dei Tarasia confessa pia devota ${ }^{45}$ ) testimonia, a mio parere, in favore dell'ipotesi di García de Castro Valdés, quella di un'esecuzione più tarda dell'epigrafe della principessa, dato che va però misurato con l'apparente condizione di umiltà della seconda Teresa, che non rendeva necessario un lungo elogio postumo, e della quale non sopravvivono ulteriori testimonianze che possano suggerirne un retroterra di alto rango.

In un recente articolo di Francisco Javier Fernández Conde ed Isabel Torrente Fernández relativo alle origini del monastero di San Pelayo di Oviedo i due autori hanno avanzato l'ipotesi che l'epigrafe funeraria di Teresa fosse stata realizzata al solo scopo di avvalorare la vicenda narrata dal vescovo Pelayo: todo hace suponer que fue redactada para justificar epigráficamente el texto cronistico pelagiano ${ }^{46}$. A mio parere, tale visione risulta possibile, ma non va assolutamente confusa con l'eventualità che l'intera esistenza di Teresa sia frutto dell'immaginazione del vescovo asturiano, come peraltro smentiscono i diplomi ad essa riconducibili di cui abbiamo trattato poco fa. Tuttavia, ritengo anche che, nel caso di una realizzazione "ad hoc" dell'epitaffio al solo scopo di giustificare quanto detto da Pelayo, sarebbe stato plausibile includere nel testo epigrafico anche una menzione della disavventura vissuta dalla

${ }^{44}$ Id., pp. 66-67.

45 Ibid.

${ }^{46}$ F. J. Fernández Conde, I. Torrente Fernández: "Los orígenes del monasterio de San Pelayo: aristocracía, poder y monacato", cit., p. I9I, nota 37; nel medesimo articolo i due autori ipotizzano che, nel caso in cui il racconto di Pelayo fosse veritiero, lo sposo di Teresa potrebbe essere stato Muhammad ibn Yayisch, morto intorno al roog e sovrano di Toledo, o suo figlio Yayisch, che regnò nella medesima città tra il Ioog ed il I035, smentendo l'ipotesi di "Abdalá" (Ibid.), oltre a negare categoricamente la possibilità che la Teresa documentata epigraficamente presso la Cattedrale di Oviedo possa essere state la figlia di Bermudo II, fatto che anch'io ritengo totalmente privo di fondamento. principessa in terra musulmana; in un contributo pubblicato anch'esso recentemente ${ }^{47}$, Laura Cayrol Bernardo è giunta a sostenere con cautela come l'effettiva presenza di Teresa all'interno del cenobio pelagiano possa essere ritenuta possibile, pur condividendo la visione "tardiva" riguardo all'epitaffio ad essa dedicato a scopi propagandistici e di prestigio (si trattava pur sempre di un' infanta regia, per giunta citata dalle cronache); certamente, si tratta di una pratica tutt'altro che assente nel mondo medievale ${ }^{48}$, ma che, se guardiamo alle pur complesse e difficilmente ricostruibili origini del monastero, aveva molte altre possibilità di applicazione, non mancando al suo interno altre figure, realmente od apparentemente, legate alla dinastia regia, con l'esempio lampante della leggenda della sua fondazione per mano del Rey Casto od addirittura di re Silo $(774-783)^{49}$.

\section{CONCLUSIONI}

Nonostante tutte le "zone d'ombra" sulla questione ed i relativi tentativi compiuti per chiarirle che ho tentato di presentare, seppur succintamente, in queste pagine, la vita di Teresa Bermúdez propone, a mio parere, una serie di punti fermi, nel mezzo della quale è possibile creare un'intelaiatura di supposizioni, ipotesi e proposte (fatte od ancora da formulare) che consentano la creazione di una "proto-biografia" della fanciulla; è quel che ho cercato di compiere in

\footnotetext{
47 L. Cayrol Bernardo, "El monasterio de San Pelayo de Oviedo: infantado y memoria regia", in Territorio, Sociedad y Poder, 8 (2013), pp. 55-66, in part. a p. 59. Sempre su tale monastero, si vedano E. Carrero Santamaría:"La ciudad santa de Oviedo, un conjunto de iglesias para la memoria del rey”, in Hortus Artium Medievalium, I3, 2007, pp. 383-384; F. J. Fernández Conde, I. Torrente Fernández: "Orígenes del monasterio de San Pelayo", in VV.AA, Semana de Historia del monacato cántabro-astur-leonés, Oviedo, 1982, pp. 99-I2I.

${ }^{48} \mathrm{Ibid}$; si vedano inoltre le pp. 6I-63 del medesimo testo, nonché, per una visione più dettagliata sul concetto di "creazione della memoria" nell'Alto Medioevo, il volume di P. J. Geary, Phantoms of Remembrance. Memory and Oblivion at the End of the First Millennium, Princeton, 1994.

49 Oltre alla tradizione che afferma come tale cenobio sia stato fondato da Alfonso II il Casto (79I-842), appaiono legate a questo sito Teresa Ansúrez, vedova di Sancho I (95I-966) e Velasquita, già moglie (in seguito ripudiata) di Bermudo II, alla quale abbiamo accennato nel testo; il già citato articolo di Laura Cayrol Bernardo evidenzia l'importanza di San Pelayo come luogo di memoria e culla dell'infantado regio.
} 
questa sede. Che si tratti di un fatto realmente accaduto, seppur dai termini molto incerti, o di un aneddoto completamente inventato, infatti, la storia del suo matrimonio con un notabile musulmano permette di gettare più luce (e spendere maggiori quantità di inchiostro, sebbene siano forse ancora troppo poche) su di una figura che, altrimenti, sarebbe indubbiamente stata dimenticata od, al massimo, relegata ad alcune righe, piatte ed incolori, a margine delle composizioni cronachistiche ed annalistiche che trattarono, nel corso dei secoli, le vite ed i regni del padre e del fratello.
Lo stesso discorso peraltro va reiterato a proposito dell'epitaffio di San Pelayo ad Oviedo, che non costituisce assolutamente una prova sicura del monacato di Teresa, ed ancor meno della su permanenza in tale cenobio, ma che non va tuttavia eliminato del tutto dal novero delle possibilità legate alla biografia di questo interessantissimo personaggio, pur dovendo l'epigrafe in questione essere -come il d'altronde frammento cronachistico che riguarda la principessa- trattata con cautela e senza ritenerla, fino a che eventuali ulteriori scoperte non lo permettano, una fonte documentaria "assoluta". 